Summary: The structural evolution of a metallocenic syndiotactic poly(propylene), sPP, with time has been studied. Due to the low syndiotactic content, the analyzed sPP sample can be easily quenched to a completely amorphous state. This amorphous sample, when left at room temperature is able to crystallize at relatively long times. The degree of crystallinity, as a function of time, has been analyzed by X-ray diffraction and DSC. The DSC melting curves show the presence of a very important low-temperature endotherm, besides the main melting peak. Real-time variable-temperature synchrotron experiments have been performed in order to analyze the origin of the two melting peaks. They seem to arise from the melting of two populations of sPP crystals with different size and/or perfection, rather than from the melting of different polymorphs. The changes in the crystalline regions are accompanied by important variations in the mechanical behavior, as revealed by DMTA and microhardness (MH) measurements. In addition, the sPP crystallization has been analyzed by MH measurements, showing the importance that a relative low crystallinity has on the stiffness of a polymeric material at temperatures above $T_{\mathrm{g}}$. On the other hand, the kinetics of the enthalpic recovery of quenched amorphous sPP specimens has been analyzed by DSC with temperature modulation. It was found that this enthalpic relaxation can be described by the KWW model, and the relaxation times and shape parameters at three different aging temperatures have been determined, with the corresponding apparent activation energy of this process.



Variation with temperature of the most probable long spacing of sPP.

\title{
Evolution of a Metallocenic sPP with Time: Changes in Crystalline Content and Enthalpic Relaxation
}

\author{
Javier Arranz-Andrés, ${ }^{1}$ Rosario Benavente, ${ }^{1}$ M. Rosário Ribeiro, ${ }^{2}$ Ernesto Pérez, ${ }^{2}$ Maria L. Cerrada ${ }^{* 1}$ \\ ${ }^{1}$ Instituto de Ciencia y Tecnología de Polímeros, C/Juan de la Cierva, 3, Madrid 28006, Spain \\ ${ }^{2}$ Grupo de Estudos de Catálise Heterogénea, Instituto Superior Técnico-ICEMS, Av. Rovisco País, Lisboa 1049-001, Portugal
}

Received: March 10, 2006; Revised: June 15, 2006; Accepted: July 6, 2006; DOI: 10.1002/macp.200600109

Keywords: crystallinity; enthalpic recovery; microhardness; $\alpha$ relaxation; syndiotactic poly(propylene)

\section{Introduction}

The structural organization and, consequently, the physical properties of polymeric materials are strongly influenced by the processing conditions such as rate of cooling, crystallization temperature, and orientation. In particular, the quenching conditions from the melt at low temperatures are very critical: as a matter of fact, temperature of quenching and time of permanence at this temperature strongly influence the phase composition and morphology. In this sense the behavior of syndiotactic poly(propylene) (sPP) is emblematic. This polymer has been known since the early work of Natta et al., ${ }^{[1,2]}$ and, recently, a highly regio- and stereoregular sPP has been synthesized with metallocene catalysts. ${ }^{[3-9]}$ The characteristics of this new sPP have been greatly improved with respect to the first polymer obtained by Natta. ${ }^{[10,11]}$ 
This polymer shows a very complex polymorphism, still in debate. ${ }^{[12-18]}$ Accordingly, great efforts have been focused on elucidating its structure. ${ }^{[19-22]}$ Four crystalline forms have been described so far and have been named as follows: ${ }^{[9,20]}$ two different orthorhombic crystalline forms denoted as Form I (ordered and disordered, both with helical conformations) and the metastable Form II, the trans-planar Form III, and the monoclinic Form IV. In addition, a mesomorphic trans-planar modification has also been reported. ${ }^{[9,23]}$

Form I is the stable structure obtained under the most common crystallization conditions either from the melt state or from solution as single crystals. Form II is an orthorhombic lattice with isochiral packing of helical chains according to the space group C222. Form III can be obtained by stretching the most stereoregular sPP samples and, as occurs with Form IV, upon solvent treatments. Recently, the crystallization of the trans-planar Form III was reported for samples kept at $0{ }^{\circ} \mathrm{C}$ for a long time without any mechanical stress ${ }^{[24]}$ though other authors have affirmed that the structure developed under those conditions do not correspond to the crystalline Form III but to a mesophase, or also paracrystalline, disordered phase. ${ }^{[23]}$ When sPP, rapidly quenched from the melt in a cold bath, is brought to room temperature it is elastic due to a very low crystallinity, but the elastic behavior is soon lost due to a relatively rapid crystallization phenomenon and to the aging of the amorphous component. ${ }^{[25]}$

Physical aging response of polymers is an active area of research due to its practical importance since it bears both on the dimensional stability of the material during processing and upon the subsequent long-term performance of a finished product. During a controlled cooling of a polymer in the equilibrium melt state from a high temperature, a moment is reached when the rate of structure rearrangement in the melt state, which depends on the thermal mobility of chain segments, starts to lag behind the cooling rate. The macromolecules in the sample will then show a progressive deviation in conformation from their equilibrium. At low temperature, the sample will possess a frozen-in structure. With time, this metastable structure will relax towards the equilibrium state, the process referred as structural recovery. This phenomenon gathered together with subsequent changes in properties (physical aging) have been reviewed by Hodge, ${ }^{[26]}$ Mijovic, ${ }^{[27]}$ and Hutchinson. ${ }^{[28]}$

DSC is one of the most often applied experimental techniques to study the glass transition, vitrification, and structural recovery in the glassy state. ${ }^{[29]}$ The development of temperature-modulated differential scanning calorimetry (TMDSC) by Reading ${ }^{[30]}$ a few years ago has greatly increased the quality of the structural information that can be obtained by calorimetry in some specific topics. In this technique, a modulation is superimposed on the conventional temperature program and a Fourier transform analysis is applied to the resultant data. The purported advantages of TMDSC include the ability to separate overlapping phenomena, as well as improved resolution and sensitivity. Then, TMDSC allows the calculation of reversing heat flow (caused by the modulation) and total heat flow (caused by the underlying heating rate and is related to conventional DSC data). The difference is the non-reversing heat flow, indicating the contribution of all the kinetics events which cannot follow the modulation. Therefore, with this technique separate information can be obtained when structural relaxation is superimposed to glass transition since the first process appears in the total and non-reversing component of the heat flow while the second event shows up in the total and reversing component of the heat flow.

The main objective of this investigation is to analyze the evolution of an initially amorphous sPP structure when it is maintained at temperatures either above or below its glass transition, $T_{\mathrm{g}}$. The great interest of this research consists of starting from a complete amorphous sPP specimen, because a partial crystallinity, organized in any of the different polymorphs, is always obtained from the common degrees of syndiotacticity found in the literature. The annealing at temperatures above $T_{\mathrm{g}}$ mainly affects the crystalline regions, as shown by X-ray diffraction and DSC. Dynamic mechanical thermal analysis, DMTA, and microhardness $(\mathrm{MH})$ are used, for the first time to the best of our knowledge, as probes of the subsequent mobility reduction associated with crystallization. However, the sPP maintenance at temperature below $T_{\mathrm{g}}$ provokes motion restrictions ascribed to the amorphous phase and the resultant enthalpic recovery is characterized by MDSC. The parameters that govern this process have been estimated assuming a Kohlrausch-Williams-Watss (KWW) behavior.

\section{Experimental Part}

An sPP was synthesized with a syndiospecific catalyst as described elsewhere. ${ }^{[31,32]}$ The homogeneous metallocene syndiospecific catalyst and cocatalyst employed were $\mathrm{Ph}_{2} \mathrm{C}(\mathrm{Flu})(\mathrm{Cp}) \mathrm{ZrCl}_{2}$ and methylaluminoxane (MAO), respectively. The characteristics of the polymer are reported in Table 1 . Syndiotacticity was determined by the analysis of the ${ }^{13} \mathrm{C}$ NMR solution spectrum at $120^{\circ} \mathrm{C}$ in $1,1,2,2$-tetrachlorodideuterioethane using hexamethyldisiloxane (HMDS) as internal chemical shift reference. The molecular weight and its distribution of this sPP were evaluated by gel permeation chromatography (GPC) using a Waters 150-C ALC/GPC instrument, equipped with four $300 \times 7.5 \mathrm{~mm}^{2}$ columns type PL GelMIXEDA20 $\mathrm{mm}$ and a Jasco $875 \mathrm{UV}$ detector set at

Table 1. Characteristics of the sPP sample analyzed.

\begin{tabular}{lcccc}
\hline Sample & rrrr & & $10^{-3} \bar{M}_{\mathrm{w}}$ & $\bar{M}_{\mathrm{w}} / \bar{M}_{\mathrm{n}}$ \\
\cline { 2 - 3 } & $\%$ & & $\mathrm{~g} \cdot \mathrm{mol}^{-1}$ & \\
\hline sPP & 63 & 130 & 1.8
\end{tabular}


$254 \mathrm{~nm}$. Polystyrene as standard and trichlorobenzene as solvent were used. The temperature of the experiment was $135^{\circ} \mathrm{C}$ with a flow rate of $1 \mathrm{ml} \cdot \mathrm{min}^{-1}$.

Films were obtained by compression molding in a Collin press between hot plates $\left(150^{\circ} \mathrm{C}\right)$ at a pressure of $2 \mathrm{MPa}$ for $4 \mathrm{~min}$, and a subsequent quenching to room temperature between plates refrigerated with water. To evaluate the evolution of the crystalline structure some films were left at room temperature for different times prior to perform their corresponding structural, thermal, and mechanical characterization. These distinct sPP specimens were labeled as: sPP- followed by $h$ or $d$ indicating hours or days, respectively, and by a number related to how many hours or days the specimen was kept at room temperature.

Wide-angle X-ray diffraction (WAXS) patterns were recorded in the reflection mode at room temperature by using a Philips diffractometer with a Geiger counter, connected to a computer. Ni-filtered $\mathrm{Cu} \mathrm{K} \alpha$ radiation was used. The diffraction scans were collected over a period of $20 \mathrm{~min}$ in the range of $2 \theta$ values from $3^{\circ}$ to $43^{\circ}$, using a sampling rate of $1 \mathrm{~Hz}$. The goniometer was calibrated with a silicon standard.

The synchrotron studies were performed in the soft-condensed matter beamline A2 at Hasylab (Hamburg, Germany), working at a wavelength of $0.150 \mathrm{~nm}$. Two different setups were used. In the first one a MAR CCD detector, at a distance of $17 \mathrm{~cm}$ from the sample (which is inside the temperature controller of the beamline) was used for studying the WAXS region. A sample of silver behenate (giving a well-defined diffraction at a spacing of $5.838 \mathrm{~nm}$, and several orders) was used for calibration. A heating rate of $8{ }^{\circ} \mathrm{C} \cdot \mathrm{min}^{-1}$ was employed, acquiring images every $15 \mathrm{~s}$. Two-dimensional X-ray patterns were processed with the FIT2D program of Dr. Hammersley (ESRF) and converted into one-dimensional arrays after normalization for the intensity of the primary beam and subtraction of the scattering of an empty sample.

In the second setup, two linear position-sensitive detectors were used simultaneously, one of them at $230 \mathrm{~cm}$ from the sample (covering the small angle scattering, SAXS, region) and the other at around $17 \mathrm{~cm}$ from the sample and covering the approximate $2 \theta$ range from $11^{\circ}$ to $30^{\circ}$ (WAXS region). This WAXS detector was calibrated with the diffractions of a crystalline PET sample, and the SAXS detector, with the different orders of the long spacing of rat-tail cornea $(L=65 \mathrm{~nm})$. A heating rate of $16 \pm \mathrm{C} \cdot \mathrm{min}^{-1}$ was used in this case, acquiring frames every $15 \mathrm{~s}$.

DSC measurements were performed to determine total the heat flow and TMDSC to estimate the complex heat capacity. On the one hand, conventional calorimetric analyses were carried out in a Perkin-Elmer DSC7 calorimeter, connected to a cooling system and calibrated with different standards. The sample weights ranged from 6 to $8 \mathrm{mg}$. A temperature range from -50 to $150{ }^{\circ} \mathrm{C}$ has been studied and the used heating rates were 20 and $40^{\circ} \mathrm{C} \mathrm{min}^{-1}$. For crystallinity determinations, a value of $196.6 \mathrm{~J} \cdot \mathrm{g}^{-1}$ has been taken as the enthalpy of fusion of a perfectly crystalline material. ${ }^{[9,33]}$ On the other hand, modulated differential scanning calorimetric experiments were performed on a TA Instruments 2920 MT-DSC calorimeter, connected to a cooling system and calibrated also with different standards. Calibration for heat flow scales was made using indium as the reference and checked via heat capacity measurements with a sapphire disk in the range of interest. The sample weights were around $11 \mathrm{mg}$ in all the experiments. MDSC experiments were performed with a sinusoidal temperature oscillation overlaid on the linear temperature ramp. Preferred modulation parameters are a period of $60 \mathrm{~s}$, an amplitude of $1{ }^{\circ} \mathrm{C}$, and an underlying heating rate of $1.5^{\circ} \mathrm{C} \cdot \mathrm{min}^{-1}$. A temperature range of -50 to $20^{\circ} \mathrm{C}$ has been studied.

Viscoelastic properties were measured with a Polymer Laboratories MK II dynamic mechanical thermal analyzer working in the tensile mode. The real $\left(E^{\prime}\right)$ and imaginary $\left(E^{\prime \prime}\right)$ components of the complex modulus and the loss tangent (tan $\delta$ ) of each sample were determined at $1,3,10$, and $30 \mathrm{~Hz}$, over a temperature range of -150 to $100{ }^{\circ} \mathrm{C}$, at a heating rate of $1.5^{\circ} \mathrm{C} \cdot \mathrm{min}^{-1}$. The specimens used were rectangular strips of $2.2 \mathrm{~mm}$ wide, around $0.7 \mathrm{~mm}$ thick, and over $15 \mathrm{~mm}$ long.

A Vickers indentor attached to a Leitz MH tester was used to carry out microindentation measurements. Experiments were undertaken from 20 to $100^{\circ} \mathrm{C}$. The temperature was raised in steps of 5 or $10^{\circ} \mathrm{C}$, allowing $3 \mathrm{~min}$ for temperature equilibration, and then performing $\mathrm{MH}$ determination. The heating control was made with a hot stage Linkam TM S 92 and an aluminum adaptor. A contact load of $0.98 \mathrm{~N}$ and a contact time of $25 \mathrm{~s}$ were employed. The experimental values are the average of several measurements. $\mathrm{MH}$ values (in $\mathrm{MPa}$ ) were calculated according to the relation: ${ }^{[34]}$

$$
\mathrm{MH}=2 \sin 68^{\circ}\left(P / d^{2}\right)
$$

where $P$ (in $\mathrm{N}$ ) is the contact load and $d$ (in $\mathrm{mm}$ ) the diagonal length of the projected indentation area.

\section{Results and Discussion}

\section{Evolution of Crystalline Structure above $T_{g}$}

Figure 1 shows the WAXS patterns of the sPP sample for different annealing times at room temperature after its fast cooling from the melt. An efficiently quenched and rapidly analyzed sPP sample, sPP-h0, is completely amorphous, as seen by the appearance of its characteristic amorphous halo similar to that exhibited by an atactic poly(propylene) ${ }^{[35]}$ (diffractogram represented at bottom of this figure). It is worth noting that preparation of amorphous $\mathrm{SPP}$ is not simple and the quenching at $0{ }^{\circ} \mathrm{C}$ induces formation of its mesomorphic form ${ }^{[36-44]}$ for specimens with relatively high syndiotacticity. The moderately low syndiotactic degree exhibited by this SPP under study delays its crystallization rate and allows its preparation in a complete amorphous state, as seen for sPP-h0 diffractogram. However, this sPP is able to crystallize if it is kept at room temperature for enough time. Therefore, the nascent crystal peaks become visible on the amorphous halo just after $1 \mathrm{~h}$ at room temperature, sPP-h1 sample. Some well-defined diffractions start clearly to emerge after remaining at this temperature above $T_{\mathrm{g}}$ for two or three hours, sPP-h2 and sPP-h3 specimens, respectively. These patterns are characteristic of the disordered Form I that consists of an orthorhombic lattice 


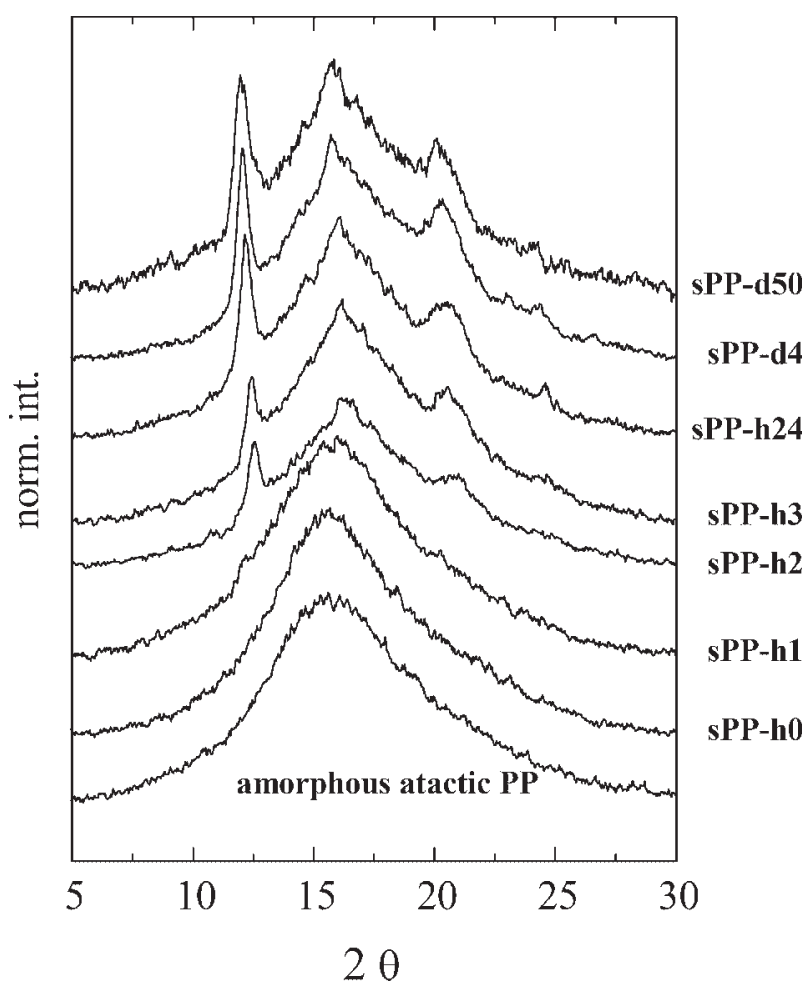

Figure 1. X-ray diffraction patterns, at room temperature, for the different samples. From top to bottom: sPP-d50, sPP-d4, sPP-h24, sPP-h3, sPP-h2, sPP-h1, sPP-h0 and a completely amorphous atactic PP.

where disorder comes from defects in the regular alternation of right- and left-handed helices along both axes of the unit cell. ${ }^{[9,21]}$ Four diffractions of this crystalline lattice are observed in the $2 \theta$ representation at around $12.2^{\circ}, 15.8^{\circ}, 20.8^{\circ}$ and a small one at 24.5 of $2 \theta$, corresponding to the (200), (010), (111), and (400) reflections, respectively. ${ }^{[9,20,21,45]} \mathrm{A}$ similar behavior has been reported for other sPP samples of low syndiotacticity. ${ }^{[8,46]}$ The absence of the reflection at $2 \theta=18.8^{\circ}$ is a clear indication that the disordered Form I is obtained, as expected because of the quench imposed during preparation of films and the low syndiotacticity of this sPP under study. For a longer time of $24 \mathrm{~h}$, sPP-h24, diffractions become sharper and better-defined indicating a higher crystallinity and more perfect crystals. This improvement in crystalline structure is additionally observed for the longest times here represented, sPP-d4 and sPP-d50 samples, and the peaks are moved to slightly lower angles. Therefore, annealing at room temperature of this sPP leads to its transformation from an entire amorphous state to other semicrystalline one with increasing crystallinity and perfection with time. The absence of sPP with annealing time of diffraction at $18.8^{\circ}$ confirms that Form I is still disordered. However, as it was also reported for sPP copolymers, ${ }^{[4]}$ it is impossible to assure the non-coexistence of this polymorph with a certain small amount of disordered modification of Form II containing kink-bands, ${ }^{[9,48]}$ appearing this latest contribution at $2 \theta$
Table 2. X-ray crystallinity and thermal characteristics estimated from the first run by DSC measurements: glass transition and melting temperatures, and DSC crystallinity.

\begin{tabular}{lccccc}
\hline Sample & $f_{\mathrm{c}}^{\mathrm{RX}}$ & \multicolumn{2}{c}{$T_{\mathrm{g}}$} & & \multicolumn{2}{c}{$T_{\mathrm{m}}$} & $f_{\mathrm{c}}^{\mathrm{DSC}}$ \\
& & ${ }^{\circ} \mathrm{C}$ & & \\
\hline sPP-h0 & 0.00 & -1 & & \\
sPP-h1 & 0.02 & -1 & & 95 & 0.00 \\
sPP-h2 & 0.10 & 0 & & 94 & 0.01 \\
sPP-h3 & 0.17 & 2 & & 93 & 0.12 \\
sPP-h24 & 0.22 & 3 & & 94 & 0.15 \\
sPP-d4 & 0.25 & 4 & & 93 & 0.16 \\
sPP-d50 & 0.30 & 5 & 93 & 0.18
\end{tabular}

around $17-18^{\circ}$. All of these changes within the crystalline regions significantly affect some other parameters such as density, ${ }^{[49]}$ physical properties ${ }^{[50]}$ and mechanical behavior, as will be discussed below.

To estimate the crystallinity from the different patterns, the amorphous halo obtained for specimen $\mathrm{sPP}-\mathrm{h} 0$ has been subtracted from those acquired from the other films maintained at room temperature for different annealing times. The values attained are listed in Table 2. It seems that the maximum crystallinity value reached for this $\mathrm{SPP}$ with a $63 \%$ syndiotacticity is only around $30 \%$, as corresponds to its moderately low syndiotactic content. ${ }^{[9]}$

The development of crystalline structure also has been followed by calorimetric measurements. Figure 2 shows conventional DSC melting curves for the SPP at different annealing times at room temperature. The data obtained for different thermal parameters are listed in Table 2. A unique thermal transition, associated with the glass transition, $T_{\mathrm{g}}$, is observed in the sample just-cooled, sPP-h0, indicating that it is completely amorphous in agreement with WAXS results. $T_{\mathrm{g}}$ is located at around $-1{ }^{\circ} \mathrm{C}$. In addition, cold crystallization during heating run and its subsequent melting are not exhibited. However, if a specimen is left for $1 \mathrm{~h}$ at room temperature after its cooling, sPP-h1, several differences appear. The first of them is ascribed to the slight decrease in the heat capacity along glass transition whereas the second one is related to the appearance of a cold crystallization process during the heating run and after that the melting of the corresponding crystallites. The first characteristic indicates the development of a very small amount of crystallinity, in agreement with the nascent diffraction peaks observed in Figure 1. Moreover, crystal nuclei have been formed during the period of stay at room temperature. The existence of these nuclei makes possible the crystallization during the heating run. These features are more significant for the specimen sPP-h2 since during its stay at room temperature some more, small, and imperfect ordered entities have been developed (see Figure 1). Accordingly, the reduction in heat capacity during glass transition is enlarged and the extension of cold crystallization during the heating run is larger. (Obviously, 


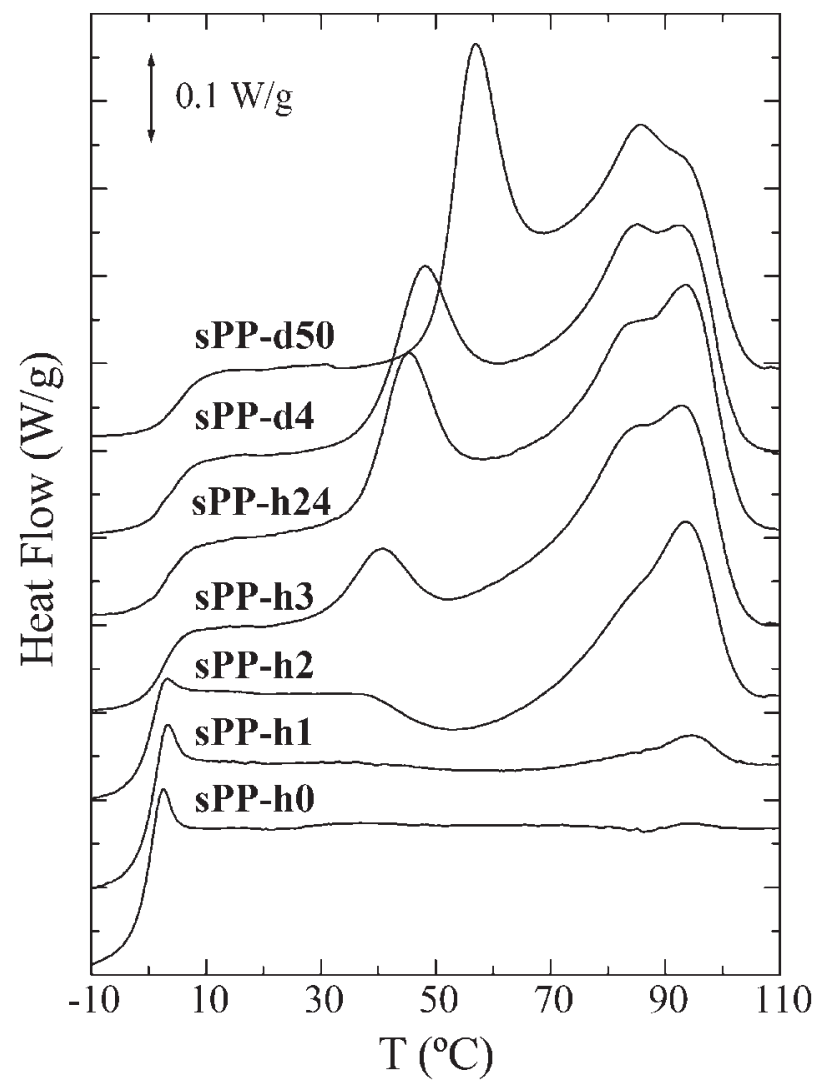

Figure 2. DSC first melting curves for the different samples. From top to bottom: sPP-d50, sPP-d4, sPP-h24, sPP-h3, sPP-h2, sPP-h1, and sPP-h0. Heating rate: $20^{\circ} \mathrm{C} \cdot \mathrm{min}^{-1}$.

crystallinity of the samples has been determined from the enthalpy obtained from just above the glass transition to the end of the final endothermic peak, so that crystallinity formed during the cold crystallization is discounted.)

It is interesting to note the existence of a small endothermic peak at the top of the glass transition for the three samples of smaller annealing time. This endothermic peak is due to the physical aging of the sample during the short residence time of the specimen at temperatures slightly below the glass transition during the heating run. When crystallinity reaches a certain value, this peak is not observed any longer (sample sPP-h3) since the constraints imposed by the crystal entities to the amorphous regions preclude the enthalpic recovery during the short residence time throughout the heating.

In spite of the very slow crystallization rate exhibited by this sPP, three hours at room temperature seems to be enough time to allow a much higher degree of crystallization because, although crystallites are rather imperfect and small, looking at WAXS pattern, a cold crystallization is no longer observed during heating. These actual crystallites impose much higher mobility constraints on the amorphous macromolecules and, consequently, an important decrease in heat capacity is observed with a shift of $T_{\mathrm{g}}$ to higher temperatures. The main melting endotherm appears at $94{ }^{\circ} \mathrm{C}$, as in sPP-h1 and sPP-h2. This rather low melting temperature is due to the relatively low syndiotactic content and causes the sample to have a considerable tail at the low temperature side of the melting curve that extends down to room temperature. Additionally, a small endotherm is observed at $40^{\circ} \mathrm{C}$. This feature might arise from the annealing process related to maintenance of the sample at room temperature, as observed in other olefinic materials, ${ }^{[47,51,52]}$ where the annealing peak appears at temperature around $15-30^{\circ}$ higher than the annealing temperature (room temperature in most cases). Alternatively, considering the complicated polymorphic behavior of sPP aforementioned, this endotherm could also be associated with a transformation (or simply melting) from a polymorph different from the major crystalline structure that melts at $94{ }^{\circ} \mathrm{C}$.

The longest annealing times, e.g., for specimens sPP-d4 and sPP-d50, lead to a reduction of heat capacity in the region of glass transition and to a displacement of its location to slightly higher temperatures. In addition, the lower melting peak is moved to higher temperature and its intensity is considerably increased with annealing time. However, crystallinity almost reaches its maximum value for this sPP in the specimen sPP-d4, and then it is kept practically constant in sPP-d50, as seen in Table 2.

In order to analyze the origin of these two melting peaks, real-time variable-temperature synchrotron experiments have been performed on this sPP sample, after several months at room temperature. In a first experiment, the WAXS region has been investigated. The diffractograms corresponding to that sample in a melting experiment are shown in Figure 3. The initial pattern is rather similar to that in Figure 1 for sample sPP-d50, but now with a much better signal-to-noise ratio. These results indicate that, as can be deduced from the two highlighted profiles in Figure 3, besides the decrease in crystallinity due to partial melting, the pattern is very similar along the experiment, until total melting, which occurs around $104^{\circ} \mathrm{C}$.

Since the (200) diffraction is rather sharp and well-isolated, its evolution with temperature can be easily analyzed. The results are presented in Figure 4. The lower part of this Figure represents the area of this diffraction as a function of temperature. A sharp decrease in its intensity is observed at around $57^{\circ} \mathrm{C}$, followed by a more smooth decrease until total disappearance at $104^{\circ} \mathrm{C}$. The derivative of this area is plotted in the middle part of Figure 4, compared with the actual DSC melting curve of the sample (which is practically identical to the DSC curve of sample sPP-d50 in Figure 2). It can be observed that there is a perfect correlation between the derivative of the area of the (200) diffraction and the melting curve. This means, most probably, that diffraction (200) is involved in all the melting processes reflected in the different melting peaks. Moreover, the "constancy" with temperature of the diffraction patterns (except for the crystallinity amount) indicates that 


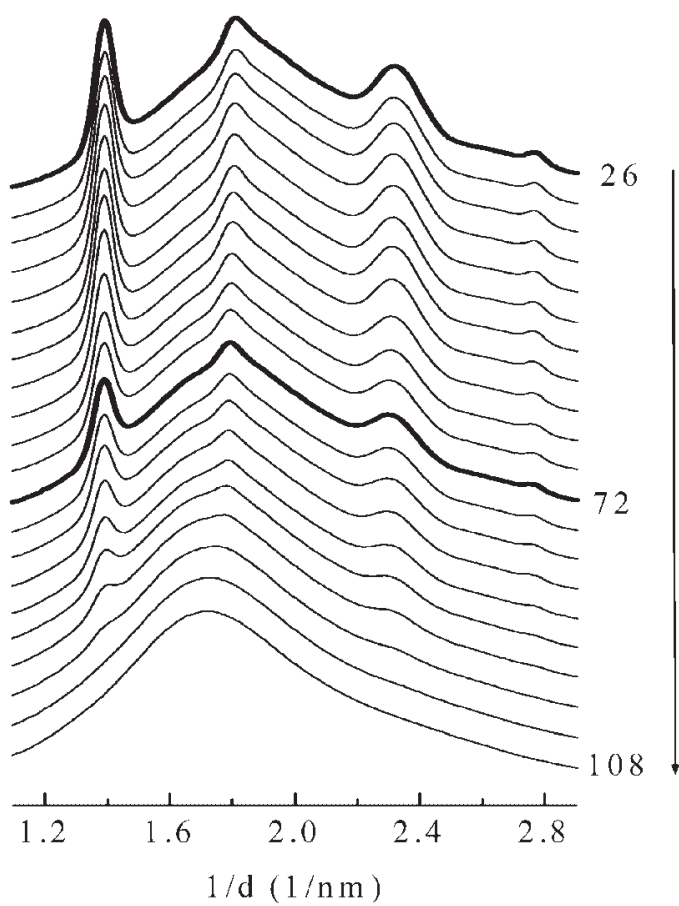

$\mathrm{T}\left({ }^{\circ} \mathrm{C}\right)$

Figure 3. Synchrotron diffraction patterns in the WAXS region recorded in a melting experiment of the sPP sample after several months at room temperature. For clarity, only one of every two frames is plotted. The patterns at room temperature and at $72{ }^{\circ} \mathrm{C}$ are highlighted.

the two melting peaks observed are not due, in principle, to preferential melting of a certain polymorph.

Additional information has been obtained from the analysis of the SAXS region. The profiles corresponding to a melting experiment are shown in Figure 5. At room temperature, a rather wide and long spacing is observed, centered at around $10 \mathrm{~nm}$. It presents a very low intensity, as it corresponds to rather imperfect crystals, with a presumably small electronic density difference between the amorphous and crystalline layers. This long spacing remains approximately constant up to around $60^{\circ} \mathrm{C}$, when its tail at higher values of $1 / d$ (lower spacings) disappears, so that the value of the most probable long spacing increases, as can be observed in Figure 6. At temperatures above $90{ }^{\circ} \mathrm{C}$, the long spacing is not observed anymore, partially because it may fall outside the detection window. It is important to note that the usual rather large increase in the area below the long spacing during melting, associated to recrystallization phenomena, is not observed here.

From all these synchrotron experiments it seems, therefore, that the two melting endotherms observed in Figure 1 are due to the melting of two populations of sPP crystals with different size and/or perfection, rather than to different polymorphs.

Anyway, the structure of this sPP sample is significantly changing with annealing time at room temperature, as evaluated by WAXS and DSC: SPP is initially completely



Figure 4. Variation with temperature, in the melting experiment of Figure 3, of the area of the (200) diffraction peak (lower part) and of the derivative of that area (middle part) compared with the DSC melting curve (upper part).

amorphous, after three hours the crystallinity is $12 \%$, and after four days it practically reaches its upper value of crystallinity. This continuous evolution of structure implies a permanent change in mechanical properties. To analyze this variation and considering the rather small crystallinity change, only the two extreme specimens have been chosen, sPP-h0 and sPP-d50.

Figure 7 shows plots of storage and loss moduli, and $\tan \delta$ as a function of temperature at $3 \mathrm{~Hz}$ for the sPP-d50 and sPP-h0 specimens. Looking first at the viscoelastic behavior exhibited by sPP-d50, it has to be said that three different relaxations are observed, labeled as $\gamma, \beta$, and $\alpha$, in the order of increasing temperature. The $\gamma$ process appears at around $-80^{\circ} \mathrm{C}$ and is ascribed to local motions of methyl groups within the skeleton in SPP. This relaxation is quite less broad than that for specimens with similar molecular origin found 


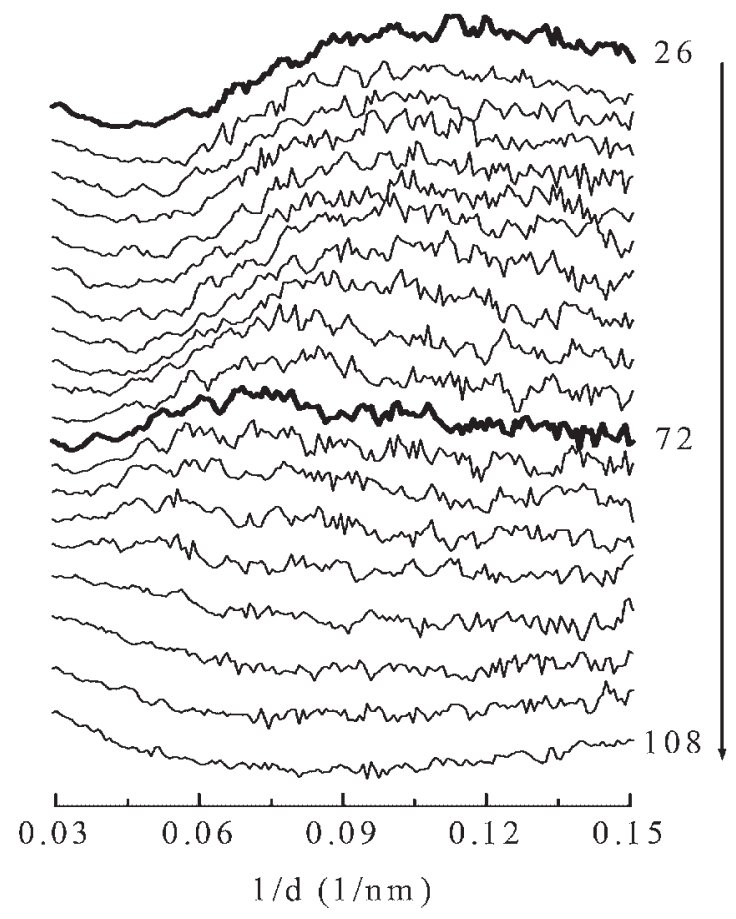

Figure 5. Lorentz-corrected SAXS profiles of sPP in a melting experiment. The profiles at room temperature and at $72{ }^{\circ} \mathrm{C}$ are highlighted.

in iPP because of the lower steric restrictions between the alternative methyl groups.

The $\beta$ relaxation is identified with the glass transition of sPP. Therefore, it is related to generalized motions within the amorphous regions. Its location is in perfect agreement with that estimated by calorimetric measurements.

There remains no clear consensus regarding the origin, and even the existence, of the $\alpha$ process, that occurs at temperatures above the relaxation temperature, related to the glass transition ( $\beta$ mechanism). There are many opinions which support the absence ${ }^{[50,53]}$ of any relaxation above the $\beta$ process for sPP. However, its presence has been recognized by some authors, ${ }^{[54,55]}$ though its origin is not clear. On the one hand, a second glass transition has been proposed $^{[54]}$ similar to what has been described by some researchers ${ }^{[56,57]}$ for the stereoisomer iPP. On the other hand, this $\alpha$ relaxation has been related to a change in the modes of force transmission or, more precisely, a change in the mechanical coupling of the force-transmitting rigid crystalline entities. ${ }^{[55]}$

Figure 7 clearly depicts this $\alpha$ relaxation in the variation of either storage or loss moduli at temperatures above $T_{\mathrm{g}}$. Previous studies ${ }^{[47]}$ have suggested a relationship with mobility within the crystalline regions combining a continuous melting of the smaller crystallites with the motion of those with a slighty larger size. Since the enthalpy associated with this melting process is significant, the loss of rigidity is also important. Its relationship with crystallites is

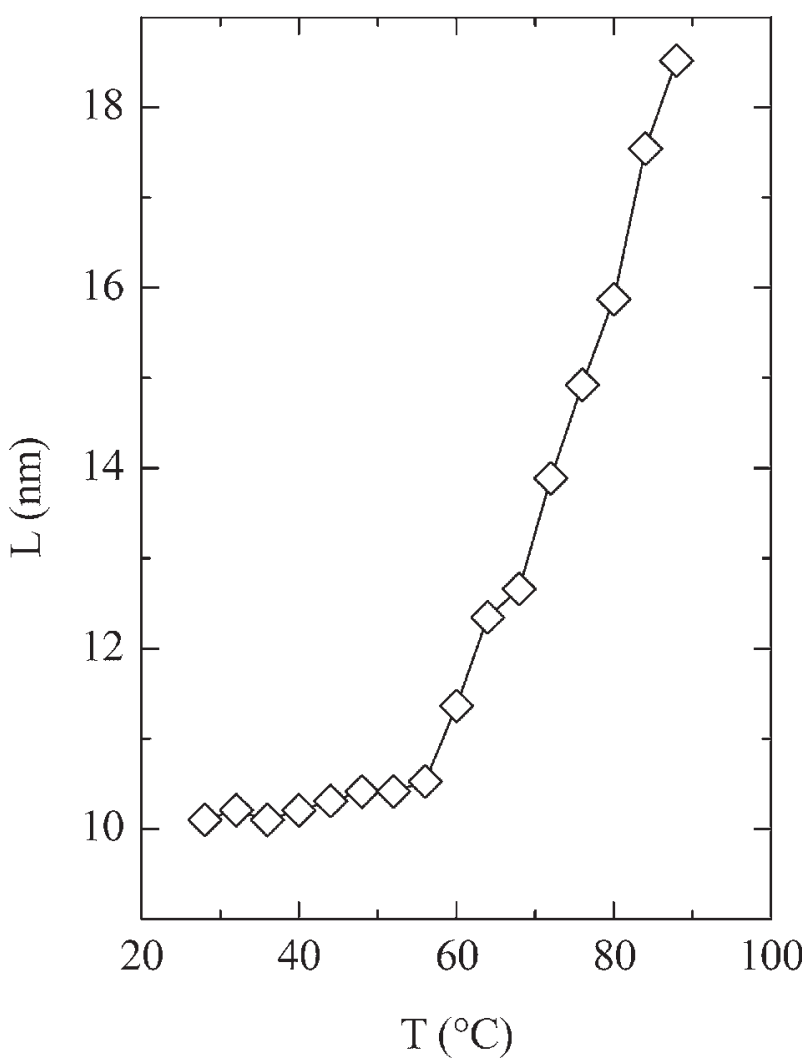

Figure 6. Variation with temperature of the most probable long spacing of sPP in the melting experiment of Figure 5.

now experimentally confirmed analyzing the viscoelastic behavior of the sample sPP-h0 completely amorphous at the beginning of the experiment. It is seen that above the $\beta$ relaxation, a significant increase in either storage or loss moduli takes place. This $\beta$ mechanism is associated with cooperative motions within amorphous regions, as mentioned above. Therefore, it is much more intense, looking at the $\tan \delta$ representation, than the same process in the sPP$\mathrm{d} 50$ because of the entire sPP-h 0 amorphousness. Once the amorphous sPP-h0 chains reach enough mobility above $T_{\mathrm{g}}$ during the dynamic experiment, their cold crystallization starts. Consequently, rigidity rises as crystallinity is increased. Above $50^{\circ} \mathrm{C}$, the melting process of the crystallites with lower sizes begins whereas the thicker crystals still keep moving. The temperature range at which this $\alpha$ relaxation occurs for sPP-d50 and sPP-h0 specimens are in perfect agreement, as represented in Figure 7. It has to be considered that the scanning rate in these DMTA experiments is $1.5^{\circ} \mathrm{C} \cdot \mathrm{min}^{-1}$, much slower than the one in the DSC measurements $\left(20^{\circ} \mathrm{C} \cdot \mathrm{min}^{-1}\right)$. Consequently, the cold crystallization shall present a higher extension in the DMTA experiments.

It is rather unusual to observe either a crystallization process or a subsequent relaxation in storage and loss moduli in a viscoelastic experiment. But it is much less frequent to perform measurements of $\mathrm{MH}$ by varying the 


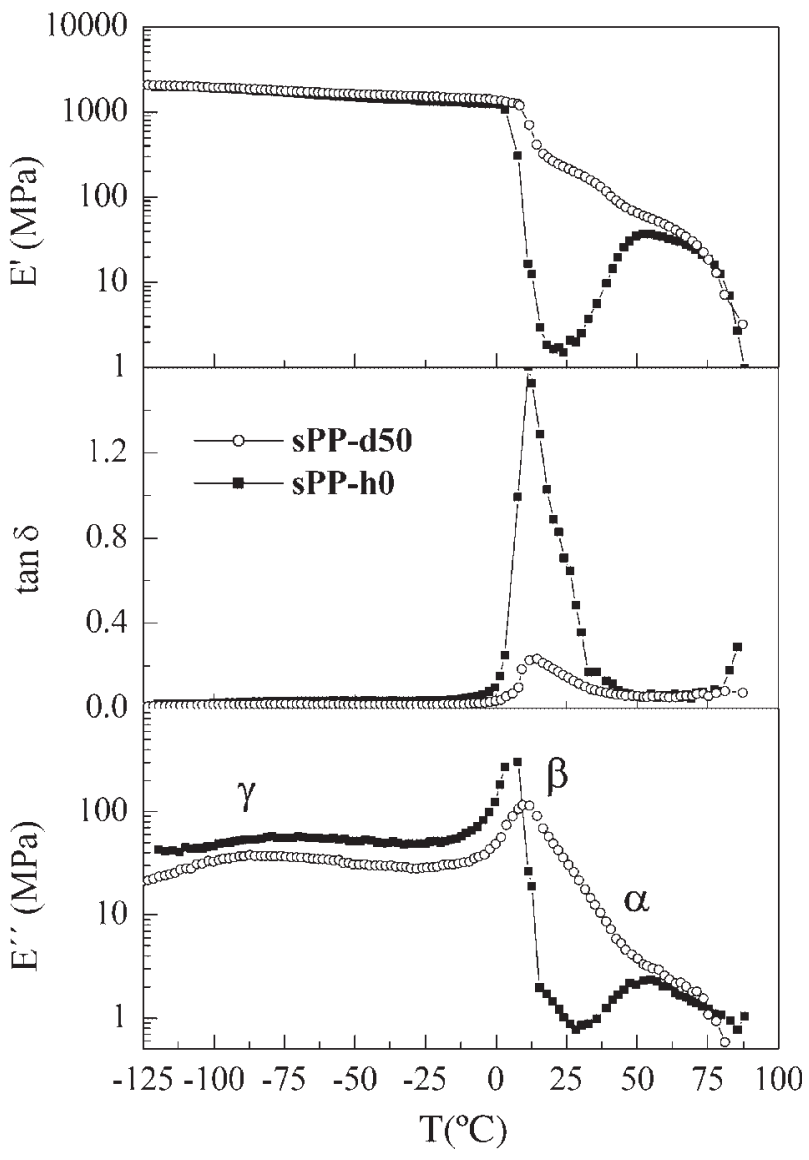

Figure 7. Temperature dependence of the real and imaginary components of complex modulus (upper and lower plot, respectively) and loss tangent (middle plot) of sPP-d50 and sPPh0.

temperature along a crystallization process. For the first time in sPP, to the best of our knowledge, we have studied the change in $\mathrm{MH}$ versus temperature for a completely amorphous and also for a partially crystallized specimen (Figure 8). In sPP-d50, similar to the variation of storage modulus, MH is continuously decreasing because the material is becoming softer as the temperature is increased. However, in sPP-h0, MH is first kept constant, but from 35 to $55^{\circ} \mathrm{C}$ it considerably increases due to the transformation of some chains from its initial amorphous state to a threedimensionally ordered state. After crystallization, $\mathrm{MH}$ values for the two specimens are analogous, as observed previously for the storage modulus.

\section{Evolution of Amorphous Structure below $T_{g}$}

To evaluate how the sPP amorphous structure evolves with time at temperatures below $T_{\mathrm{g}}$, the sPP-h0 specimen has been selected to avoid the interference of the crystalline entities along the structural relaxation. As commented in the Introduction, at temperatures below $T_{\mathrm{g}}$, the sample possesses a metastable frozen-in structure that relaxes with

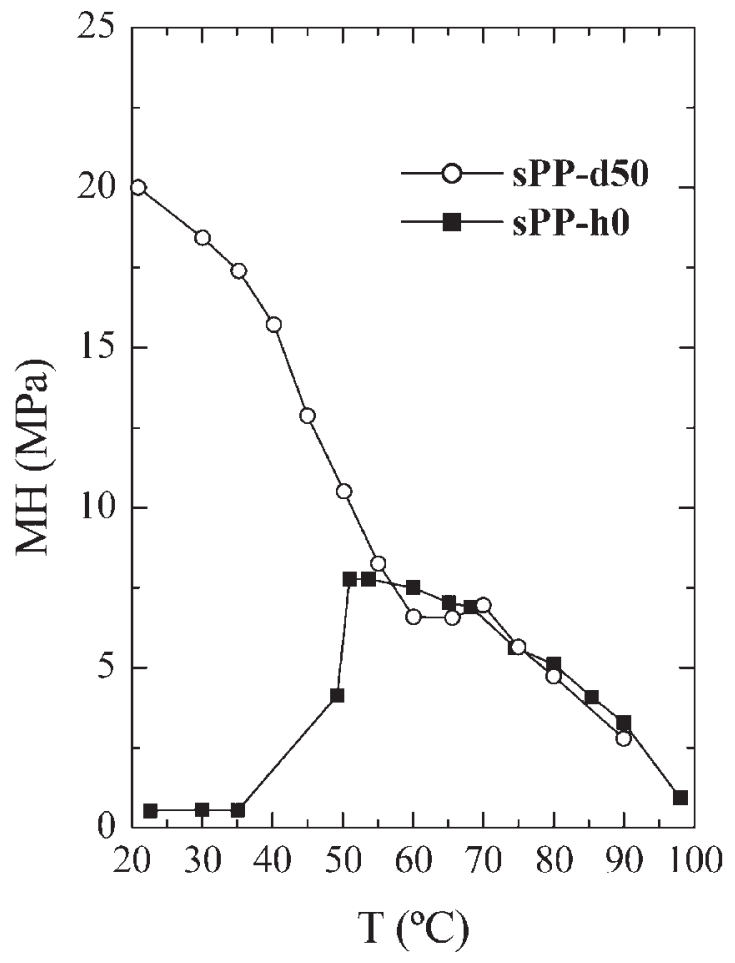

Figure 8. Variation of $\mathrm{MH}$, with temperature for sPP-d50 and sPP-h0.

time towards its equilibrium state. In the current investigation, enthalpy is the thermodynamic quantity chosen to be followed. Therefore, the structural recovery under study can be referred to as enthalpic recovery. Its evolution with time can be described by different models ${ }^{[26,28,58,59]}$ and one of these is based on the Kohlrausch-Williams-Watts $(\mathrm{KWW})$ model behavior for the relaxation function that uses a stretched exponential response function given by:

$$
\Phi=\exp \left[-\left(t_{a} / \tau\right)^{\beta}\right]
$$

where $\Phi$ is the relaxation function, $t_{\mathrm{a}}$ the aging time, $\tau$ the effective relaxation time, and $\beta$ the shape parameter which is characterizing the non-exponentiality of the system dynamics. The exponent $\beta(0<\beta<1)$ is inversely proportional to the width of the corresponding distribution of relaxation times.

The kinetics of the enthalpic relaxation process was carried out at three distinct aging temperatures $(-15,-10$, and $-5^{\circ} \mathrm{C}$ ) and different aging times, using modulated differential scanning calorimetry (MDSC) from the nonreversing heat capacity component, as shown in Figure 9 for sPP-h0 at $-10^{\circ} \mathrm{C}$. It is clearly observed that the enthalpy involved increases and the maximum of the peak is slightly shifted to higher temperatures as the aging time does. The same experiments were also performed at -5 and $-15^{\circ} \mathrm{C}$. From the area of the different curves obtained at each aging time, the corresponding enthalpic relaxation values are estimated. 


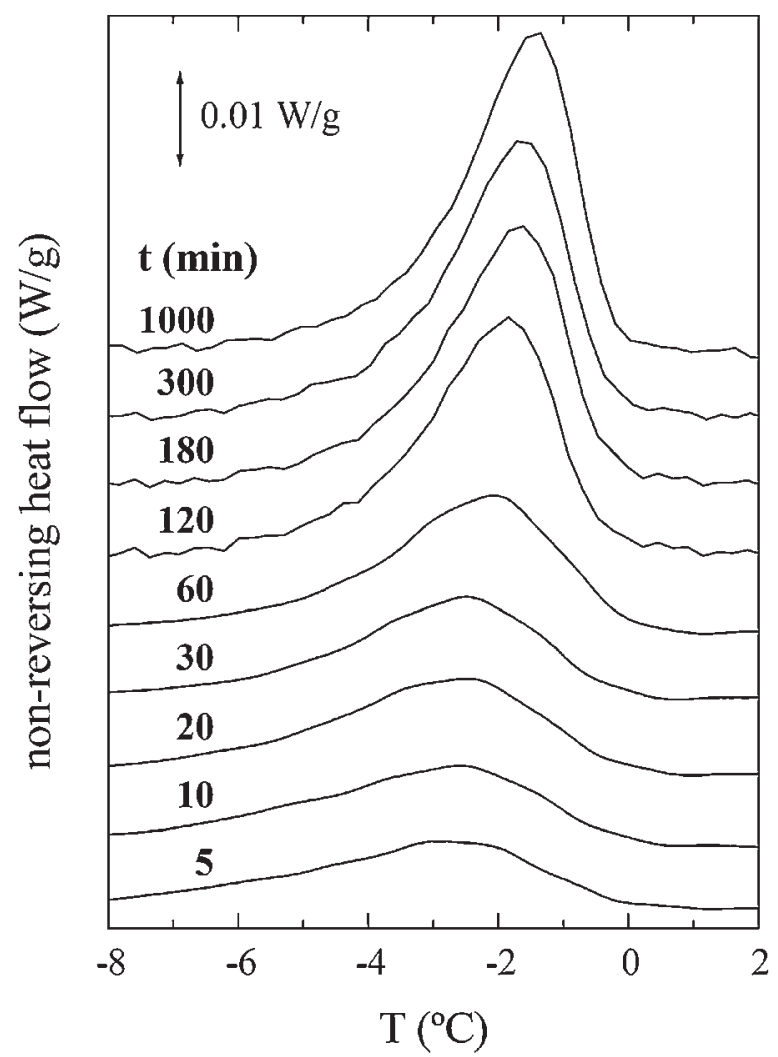

Figure 9. Dependence of non-reversing heat capacity with temperature for $\mathrm{sPP}-\mathrm{h} 0$ at different aging times at $-10^{\circ} \mathrm{C}$.

The enthalpy variation toward its equilibrium state can be represented in terms of the expression:

$$
\Delta H\left(t_{\mathrm{a}}, T_{\mathrm{a}}\right)=\Delta H\left(\infty, T_{\mathrm{a}}\right)(1-\Phi)
$$

where $\Delta H\left(t_{\mathrm{a}}, T_{\mathrm{a}}\right)$ is the measured enthalpic change after the polymer has been aged for a time $t_{\mathrm{a}}$ at an aging temperature $T_{\mathrm{a}}$, and $\Delta H\left(\infty, T_{\mathrm{a}}\right)$ is the asymptotic value of $\Delta H\left(t_{\mathrm{a}}, T_{\mathrm{a}}\right)$. It has been suggested ${ }^{[60]}$ that a linear relationship exists between this asymptotic value of heat absorption and the difference $\left(T_{\mathrm{g}}-T_{\mathrm{a}}\right)$ :

$$
\Delta H\left(\infty, T_{\mathrm{a}}\right)=\Delta c_{\mathrm{p}}\left(T_{\mathrm{g}}-T_{\mathrm{a}}\right)
$$

where $\Delta c_{\mathrm{p}}$ is the specific heat increment at the glass transition. This approximation will be valid for temperatures not very far away from $T_{\mathrm{g}}$.

Figure 10 presents the enthalpy evolution at different aging temperatures as a function of the aging time. These results have been fitted to the KWW model [combination of Equation (2) and (3)], and the effective relaxation time $\tau$, the shape parameter $\beta$, and the corresponding asymptotic value $\Delta H\left(\infty, T_{\mathrm{a}}\right)$ were determined for each annealing temperature (see Table 3 ). The values obtained for these parameters are in agreement with those obtained for a completely amorphous PP. ${ }^{[61]}$ The higher is the aging temperature, the smaller is the relaxation time for the enthalpic

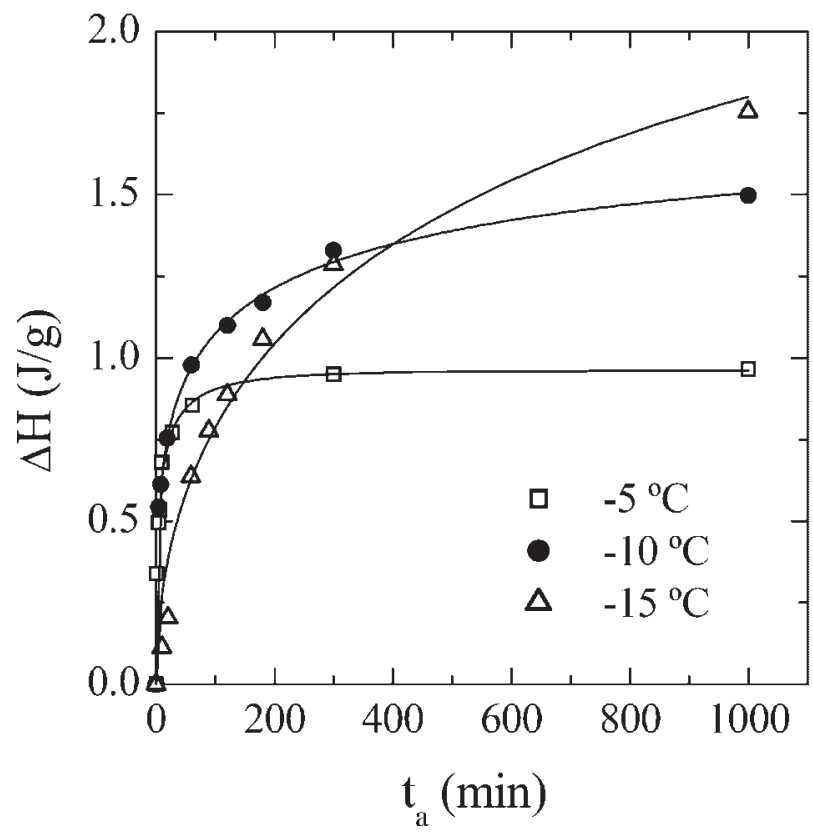

Figure 10. Change of enthalpy with aging time at the three different aging temperatures analyzed. Lines represent the KWW fits at each temperature.

recovery found, since mobility is favored by the higher temperature. Thus, this process is considerably slower at -15 than at $-5^{\circ} \mathrm{C}$. At aging temperature close to $T_{\mathrm{g}}$, the enthalpy evolution is almost completed and, then, the results are easily fitted by Equation (3). At lower aging temperatures, the enthalpic relaxation is significantly delayed, becoming a slow process for the time scale of the calorimeter. Accordingly, to get values close to the asymptotic enthalpy is almost unviable, because of the large liquid nitrogen requirements for performing measurements in the MDSC equipment used at the desired temperature range. Then, the asymptotic value at an aging temperature of $-15^{\circ} \mathrm{C}$ is still far away from that reached at the highest aging time measured (1000 min).

In the aging temperature interval studied, a very small variation is observed in the shape parameter $\beta$, indicating a similar evolution of the amorphous structure during the enthalpic relaxation at the different temperatures.

The apparent activation energy of the relaxation process, $E_{\text {app }}$, estimated by means of the expression $\tau=\tau_{0} \exp \left(E_{\text {app }} /\right.$ $R T_{\mathrm{a}}$ ), shows a value of about $190 \pm 70 \mathrm{~kJ}$. A similar activation

Table 3. Relaxation times and shape parameters for the recovery

\begin{tabular}{|c|c|c|c|}
\hline$T_{\mathrm{a}}$ & $\tau$ & $\beta$ & $\Delta H\left(\infty, T_{\mathrm{a}}\right)$ \\
\hline${ }^{\circ} \mathrm{C}$ & $\min$ & & $\mathrm{J} \cdot \mathrm{g}^{-1}$ \\
\hline $\begin{array}{l}-5 \\
-10 \\
-15\end{array}$ & $\begin{array}{c}8 \pm 1 \\
120 \pm 10 \\
215 \pm 20\end{array}$ & $\begin{array}{l}0.40 \pm 0.08 \\
0.40 \pm 0.08 \\
0.49 \pm 0.08\end{array}$ & $\begin{array}{c}0.97 \pm 0.05 \\
1.5 \pm 0.1 \\
2.5 \pm 0.2\end{array}$ \\
\hline
\end{tabular}
process fitted to a KWW model. 
energy $(270 \mathrm{~kJ})$ was found for the completely amorphous $\mathrm{PP},{ }^{[61]}$ and analogous values have been published for atactic polystyrene. ${ }^{[60]}$ Higher values, close to $960 \mathrm{~kJ}$, are reported for amorphous polymers with more rigid structures like $\mathrm{PET},{ }^{[62]}$ while an $E_{\mathrm{app}} \approx 96 \mathrm{~kJ}$ controls the evolution of the liquid crystal polyester system poly(diethylene glycol $p, p^{\prime}$ bibenzoate). ${ }^{[63]}$

\section{Conclusion}

Investigation of the crystalline structure of an sPP homopolymer with relatively low syndiotacticity has pointed out the very low crystallization rate exhibited by this polymer. Accordingly, it is possible to obtain it in a completely amorphous state, sPP-h0 specimen, whose structure does not evolve during its heating in calorimetric measurements. This amorphousness is almost maintained in samples left for $2 \mathrm{~h}$ at room temperature, as revealed by WAXS and DSC results for sPP-h1 and sPP-h2. However, a cold crystallization process is observed in these two samples, during their subsequent DSC heating run, increasing its importance as the annealing time at room temperature rises. Longer annealing times at room temperature lead to the development of ordered entities primarily organized in the disordered Form I polymorph. The crystallinity degree attained increases as the annealing time is increased up to an upper limit of around 30\% (X-ray determination).

The DSC melting curves show the presence of a very important low-temperature endotherm, besides the main melting peak. Real-time variable-temperature synchrotron experiments have been performed to analyze the origin of the two melting peaks. They seem to arise from the melting of two populations of sPP crystals with different size and/or perfection, rather than from the melting of different polymorphs.

The changes in the crystalline regions are accompanied by important variations in the mechanical behavior. Thus, the DMTA analysis of the amorphous sPP-h0 sample shows that at temperatures above the $\beta$ mechanism, associated with the glass transition, a deep drop in $E^{\prime}$ and $E^{\prime \prime}$ occurs, followed, at temperatures slightly higher, by a significant rise of both mechanical magnitudes, ascribed to the crystallization process that is taking place within the initially amorphous SPP specimen. The $E^{\prime}$ increase is related to the higher rigidity within the polymeric material after its three-dimensional organization, whereas that associated with $E^{\prime \prime}$ concern to a relaxation that implies mobility of the crystallites just developed.

Comparing these results with those found for the completely crystallized sPP-d50 specimen, it follows that there is an unambiguous relationship between the existence of crystalline regions and the observation of the $\alpha$ relaxation, confirming, therefore, that this relaxation process arises from movements in the crystalline regions. In addi- tion, for the first time to our knowledge, the sPP crystallization has been analyzed by $\mathrm{MH}$ measurements, showing the importance that a relatively low crystallinity has on the stiffness of a polymeric material at temperatures above $T_{\mathrm{g}}$.

On the other hand, concerning the amorphous structure evolution at temperatures below $T_{\mathrm{g}}$, it can be said that the kinetics of enthalpic recovery within sPP-h0 can be perfectly described by the KWW model. In addition, relaxation times for this recovery process decrease as aging temperature increases, whereas the shape parameter $\beta$ slightly varies, indicating a similar evolution of the amorphous structure during the structural recovery at the different aging temperatures examined. The apparent activation energy for this enthalpic relaxation presents a value of about $190 \pm 70 \mathrm{~kJ}$, similar to those found for some other amorphous polymers.

Acknowledgements: The authors are grateful for the financial support of Comunidad Autónoma de Madrid (project GR/MAT/0728/ 2004), Ministerio de Educación y Ciencia (project MAT2005-00228), and Exchange Collaboration Program CSIC/GRICES (projects 2005PT0033 and Proc. 4-1-1 Espanha 2006/2007, respectively). The support from the European Commission (COST Action D17, WG D17/0004/00) is also acknowledged. The synchrotron work was supported by the European Community-Research Infrastructure Action under the FP6 "Structuring the European Research Area" Programme (through the Integrated Infrastructure Initiative "Integrating Activity on Synchrotron and Free Electron Laser Science"), contract no. RII3-CT-2004-506008 (IA-SFS). We thank the collaboration of the Hasylab personnel in the soft-condensed matter beamline A2, especially Dr. S. S. Funari.

[1] G. Natta, G. Mazzanti, G. Crespi, G. Moraglio, Chim. Ind. 1957, 39, 275.

[2] G. Natta, J. Polym. Sci. 1959, 34, 531 .

[3] J. A. Ewen, J. A. Jones, A. Razavi, J. D. Ferrara, J. Am. Chem. Soc. 1988, 110, 6255.

[4] P. Longo, A. Proto, A. Grassi, P. Ammendola, Macromolecules 1992, 24, 462.

[5] F. Grisi, P. Longo, A. Zambelli, J. A. Ewen, J. Mol. Catal. A: Chem. 1999, 140, 225.

[6] T. Shiomura, N. Uchikawa, T. Asanuma, R. Sugimoto, I. Fujio, S. Kimura, S. Harima, M. Akiyama, M. Kohno, N. Inoue, "Metallocene Based Polyolefins: Preparation, Properties and Technology", J. Scheirs, W. Kaminsky, Eds., Wiley, Chichester, England 1999, Vol. 1, pp. 437-466.

[7] J. C. Haylock, R. A. Phillips, M. D. Wolkowicz, "Metallocene Based Polyolefins: Preparation, Properties and Technology", J. Scheirs, W. Kaminsky, Eds., Wiley, Chichester, England 2000, Vol. 2, pp. 333-363.

[8] C. De Rosa, F. Auriemma, O. Ruiz de Ballesteros, L. Resconi, A. Fait, E. Claccia, I. Camurati, J. Am. Chem. Soc. 2003, 125, 10913.

[9] C. De Rosa, F. Auriemma, Prog. Polym. Sci. 2006, 31, 145.

[10] G. Natta, P. Corradini, P. Ganis, Makromol. Chem. 1960, 39, 238. 
[11] G. Natta, M. Peraldo, G. Allegra, Makromol. Chem. 1964, 75,215

[12] B. Lotz, A. J. Lovinger, R. E. Cais, Macromolecules 1988, 21,2375

[13] A. J. Lovinger, B. Lotz, D. Davis, Polymer 1990, 31, 2253.

[14] A. J. Lovinger, D. D. Davis, B. Lotz, Macromolecules 1991, 24, 552.

[15] A. J. Lovinger, B. Lotz, D. D. Davis, F. J. Padden, Macromolecules 1993, 26, 3494.

[16] Y. Chatani, H. Maruyama, K. Noguchi, T. Asanuma, T. Shiomura, J. Polym. Sci., Part C 1990, 28, 393.

[17] Y. Chatani, H. Maruyama, T. Asanuma, T. J. Shiomura, J. Polym. Sci., Part B: Polym. Phys. 1991, 29, 1649.

[18] C. De Rosa, P. Corradini, Macromolecules 1993, 26, 5719.

[19] F. Auriemma, R. H. Lewis, H. W. Spiess, C. De Rosa, Macromol. Chem. Phys. 1995, 196, 4011.

[20] C. De Rosa, F. Auriemma, P. Corradini, Macromolecules 1996, 29, 7452.

[21] C. De Rosa, F. Auriemma, V. Vinti, Macromolecules 1997, 30, 4137.

[22] C. De Rosa, F. Auriemma, V. Vinti, Macromolecules 1998, 31,7430 .

[23] V. Vittoria, L. Guadagno, A. Comotti, R. Simonutti, F. Auriemma, C. De Rosa, Macromolecules 2000, 33, 6200.

[24] T. Nakaoki, Y. Ohira, H. Hayashi, F. Horii, Macromolecules 1998, 31, 2705.

[25] L. Guadagno, C. Fontanella, V. Vittoria, P. Longo. J. Polym. Sci., Phys. Ed. 1999, 37, 173.

[26] I. M. Hodge. J. Non-Cryst. Solids 1994, 169, 211.

[27] J. Mijovic. Polym. Eng. Sci. 1994, 34, 381.

[28] J. M. Hutchinson. Prog. Polym. Sci. 1995, 20, 703.

[29] H. Suga, M. Oguni, "Chemical Dynamics: Chemistry for the 21th Century (IUPAC)", T. M. Letcher, Ed., Blackwell Science, Oxford 1999, p. 227.

[30] M. Reading, Trends Polym. Sci. 1993, 1, 248.

[31] J. L. Guevara, Ph.D. Thesis, University of Chile 2004.

[32] R. Quijada, J. L. Guevara, G. B. Galland, F. M. Rabagliati, J. M. López-Majada, Polymer 2005, 46, 1567.

[33] S. Haftka, K. Könnecke, J. Macromol. Sci. Phys. 1991, B30, 319.

[34] F. Baltá Calleja, J. Adv. Polym. Sci. 1985, 66, 117.

[35] S. Mansel, E. Pérez, R. Benavente, J. M. Pereña, A. Bello, W. Röll, R. Kirsten, S. Beck, H.-H. Brintzinger, Macromol. Chem. Phys. 1999, 200, 1292.

[36] V. Vittoria, L. Guadagno, A. Comotti, R. Simonutti, F. Auriemma, C. De Rosa, Macromolecules 2000, 33, 6200.

[37] T. Nakaoki, Y. Ohira, H. Hayashi, F. Horii, Macromolecules 1998, 31, 2705.

[38] Y. Ohira, F. Horii, T. Nakaoki, Macromolecules 2000, 33, 1801 .
[39] T. Nakaoki, T. Yamanaka, Y. Ohira, F. Horii, Macromolecules 2000, 33, 2718.

[40] Y. Ohira, F. Horii, T. Nakaoki, Macromolecules 2000, 33, 5566.

[41] Y. Ohira, F. Horii, T. Nakaoki, Macromolecules 2001, 34, 1655.

[42] T. Nakaoki, Y. Ohira, F. Horii, Polymer 2001, 42, 4555.

[43] C. De Rosa, F. Auriemma, O. Ruiz de Ballesteros, Polymer 2001, 42, 9729.

[44] C. De Rosa, O. Ruiz de Ballesteros, M. Santoro, F. Auriemma, Polymer 2003, 44, 6267.

[45] C. De Rosa, P. Corradini, Macromolecules 1993, 26, 5711.

[46] C. De Rosa, F. Auriemma, O. Ruiz de Ballesteros, Macromolecules 2004, 37, 1422.

[47] J. Arranz-Andrés, J. L. Guevara, T. Velilla, R. Quijada, R. Benavente, E. Pérez, M. L. Cerrada, Polymer 2005, 46, 12287.

[48] F. Auriemma, C. De Rosa, O. Ruiz de Ballesteros, P. Corradini, Macromolecules 1997, 30, 6586.

[49] W. R. Wheat, "Metallocene-catalyzed polymers: Materials, Properties, Processing and Markets", G. M. Benedikt, B. L. Goodall, Eds., Plastics Design Library, Norwich 1998, p. 163.

[50] I. Schwarz, M. Stranz, M. Bonnet, J. Petermann, Colloid Polym. Sci. 2001, 279, 506.

[51] A. Alizadeh, L. Richardson, J. Xu, S. McCartney, H. Marand, Y. W. Cheung, S. Chum, Macromolecules 1999, 32, 6221.

[52] M. L. Cerrada, R. Benavente, E. Pérez, Macromol. Chem. Phys. 2002, 203, 718.

[53] Y. Sakata, A. P. Unwin, I. M. Ward, J. Mater. Sci. 1995, 30, 5841.

[54] L. Guadagno, L. D’Arienzo, V. Vittoria, J. Macromol. Sci, Phys. 2000, B39, 425.

[55] Y. Men, G. Strobl, Polymer 2002, 43, 2761.

[56] R. F. Boyer, J. Macromol. Sci, Phys. 1973, B8, 503.

[57] B. Wunderlich, J. Grebowicz, Adv. Polym. Sci. 1984, 60, 1.

[58] O. S. Narayanaswamy, J. Am. Ceram. Soc. 1971, 54, 491.

[59] G. B. McKenna, "Glass Formation and Glassy Behavior", in: Comprehensive Polymer Science, Vol. 2. Polymer Properties, C. Booth, C. Price, Eds., Pergamon, Oxford, England 1989, pp. 311-363.

[60] S. E. B. Petrie, J. Polym. Sci. 1972, A2, 1255.

[61] S. Mansel, E. Pérez, R. Benavente (unpublished results).

[62] S. Montserrat, P. Cortés, Makromol. Chem., Macromol. Symp. 1988, 20, 389.

[63] V. Lorenzo, J. M. Pereña, E. Pérez, R. Benavente, A. Bello, J. Mater. Sci. 1997, 32, 3601. 